\title{
Retrospective Analysis of Prostate Cancer Recurrence Potential With Tissue Metabolomic Profiles
}

\author{
Andreas Maxeiner ${ }^{1,2}$, Christen B. Adkins ${ }^{1}$, Yifen Zhang ${ }^{1}$, Matthias Taupitz ${ }^{2}$, Elkan F. \\ Halpern ${ }^{3}$, W. Scott McDougal ${ }^{4}$, Chin-Lee Wu ${ }^{1,4}$, and Leo L. Cheng ${ }^{1,3,{ }^{*}}$ \\ ${ }^{1}$ Department of Pathology, Massachusetts General Hospital, Harvard Medical School, Boston, \\ Massachusetts \\ ${ }^{2}$ Department of Radiology, Charité Universitaetsmedizin Berlin, Berlin, Germany \\ ${ }^{3}$ Department of Radiology, Massachusetts General Hospital, Harvard Medical School, Boston, \\ Massachusetts \\ ${ }^{4}$ Department of Urology, Massachusetts General Hospital, Harvard Medical School, Boston, \\ Massachusetts
}

\begin{abstract}
BACKGROUND-In clinical care of prostate cancer patients, an improved method to assess the risk of recurrence after surgical treatment is urgently needed. We aim to retrospectively evaluate the ability of ex vivo tissue magnetic-resonance-spectroscopy-based metabolomic profiles to estimate the risk of recurrence.
\end{abstract}

METHODS-PCa recurrence is defined biochemically as the detection of serum PSA after radical prostatectomy. Sixteen consecutive PCa-recurrent cases, those with an initial PSA increase of 0.69 $\pm 0.26 \mathrm{ng} / \mathrm{ml}$ monitored $47.7 \pm 2.6$ months after prostatectomy were paired by age and Gleason score with cases without recurrence of the same pathological and clinical stages $(\mathrm{n}=16 / \mathrm{each})$. We analyzed ex vivo intact-tissue spectroscopy results from these 48 individuals at the time of prostatectomy at 14T. From these spectra, we identified the 27 most common and intense spectral metabolic regions for statistical analyses.

RESULTS-Principal component analysis (PCA) on these spectral regions from cases of clinicalstage-matched groups with and without recurrence identified four pathology-related principal components. Canonical analysis of these four and the first nine principal components for cases in the two groups defined metabolomic profiles as the canonical score that can differentiate the two groups with statistical significance. By applying the coefficients from PCA and canonical analysis to the pathological-stage-matched groups, recurrence was predicted with an accuracy of $78 \%$.

CONCLUSIONS-Results indicate the potential of tissue metabolomic profiles measured with ex vivo spectroscopy to identify PCa aggressiveness in terms of cancer recurrence. With further study, this may greatly contribute to the future design of clinical strategy for personalized treatment of PCa patients.

\section{Keywords}

high-resolution magic angle spinning; magnetic resonance spectroscopy; prostatectomy; prostate cancer recurrence

\footnotetext{
(C) 2009 Wiley-Liss, Inc.

"Correspondence to: Leo L. Cheng, PhD, Pathology Research CNY-7, 149 13th Street, Charlestown, MA 02129. cheng@nmr.mgh.harvard.edu.
} 


\section{INTRODUCTION}

Prostate cancer (PCa) is the most frequently diagnosed malignant disease of adult males, and the second leading cause of cancer death in men after lung cancer [1]. Currently, patients are screened by serum PSA test followed by biopsy [2,3]. Results of a positive PCa biopsy are incorporated into evaluations according to a series of nomograms to predict extracapsular extension, seminal vesicle invasion, and lymph node metastasis [4,5]. In addition, based on patient preoperative serum PSA level, Gleason grades, clinical stage, and other clinical conditions, empirical preoperative nomograms have also been introduced for the prediction of PCa recurrence during a 60-month period after prostatectomy [6-9]. However, information from evaluations of these preoperative nomograms becomes less significant after prostatectomy when a TNM pathology stage is defined from examination of a removed prostate and lymph nodes that dictates the nature and level of necessary post-surgery therapy $[10,11]$.

After prostatectomy, serum PSA levels of patients are tested at periodic intervals to monitor and evaluate treatment response and detect disease recurrence known as biochemical recurrence (BCR) or biochemical failure, which is defined as a detectable serum PSA level after nadir [12]. Clinically, there is a need to identify new methods which can be used in conjunction with current clinicopathological information to predict the potential of BCR after radical prostatectomy. For this reason, we designed this current retrospective study to evaluate the potential utility of metabolomic profiles for predicting BCR measured with intact-tissue magnetic resonance spectroscopy (MRS) at the time of prostatectomy for patients with BCR in comparison with prostate tissue of their matched recurrence-free patients during the same or longer durations after prostatectomy.

\section{MATERIALS AND METHODS}

\section{Patient Population and Sample Collection}

Cases evaluated in this study were among the 183 prostatectomy cases that we analyzed with intact tissue MRS between 2002 and 2003. Of these cases, 16 patients experienced $\mathrm{BCR}$, defined as an increase of the serum PSA level to the detectable level of $>0.2 \mathrm{ng} / \mathrm{ml}$ and confirmed by subsequent tests.

As a retrospective study of prostatectomy cases for which multiple samples from a single case might have been analyzed at the time of specimen acquisition, among these $16 \mathrm{BCR}$ cases we found a total of 27 independent spectroscopy and quantitative pathology results. In order to conduct pair-matched evaluations, spectroscopy and pathology values from multiple samples of a single case were averaged. To match with these 16 BCR cases, we randomly selected 32 cases without recurrence from the entire analyzed patient pool of 183 cases. These 32 age-, Gleason score-, and observation period-matched cases formed two control groups (16 cases in each) that pair with the $16 \mathrm{BCR}$ cases based on either clinical or pathological stages. Similar to the BCR cases, these control results consisting of analyses of 22 and 30 individual samples for clinical and pathological stages, respectively, were averaged for individual cases when applicable. Clinical stages included T1 and T2, while pathological stages varied from T2N0 to T3aN1. Detailed patient data are listed in Table I.

\section{MRS Was Performed With High-Resolution Magic Angle Spinning Proton Magnetic Resonance Spectroscopy (HRMAS ${ }^{1}$ HMRS)}

Here we would like to demonstrate again that high-resolution magic angle spinning (HRMAS) proton $\left({ }^{1} \mathrm{H}\right)$ MRS allows us to investigate a metabolomic analysis of still intact prostate tissue obtained by prostate needle biopsy. Its ability to study chemical metabolites in their native states shows several improvements over tedious chemical extraction 
procedures that can destroy tissue structure. All 79 samples from these 48 cases were analyzed with HRMAS ${ }^{1}$ HMRS followed by quantitative pathology as previously reported [13]. Briefly, an AVANCE system (Bruker Biospin, Billerica, MA) operating at $600 \mathrm{MHz}$ $(14.1 \mathrm{~T})$ was used for the spectroscopic measurements. A 4-mm rotor was used with Keel-F inserts to create a 10- $\mu 1$ spherical space that confines tissue at the center of the receiving coil. Two microliters of $\mathrm{D}_{2} \mathrm{O}$ was added to the tissue samples for field locking. Spectra were recorded at $4{ }^{\circ} \mathrm{C}$ with resonance frequency centered on the water resonance, a rotor synchronized DANTE experimental protocol, and spinning at 600 and $700 \mathrm{~Hz}( \pm 1.0 \mathrm{~Hz})$ [14]. A repetition time of $5 \mathrm{sec}$ and an average of 32 transients were used.

Spectroscopic data were processed with commercial software NUTS (AcornNMR, Livermore, CA) according to the following procedure: $1 \mathrm{~Hz}$ apodization before Fourier transformation, baseline correction, and phase adjustment. Resonance intensities used in this study were integrals of curve fittings with Lorentzian-Gaussian line shapes measured from either 600 or $700 \mathrm{~Hz}$ HRMAS spectra [14]. Spectral intensities analyzed in this study were relative intensities normalized by the intensity of the spectral region between 0.5 and 4.5 ppm.

\section{Quantitative Pathology}

Following spectroscopy, samples were fixed in $10 \%$ formalin, embedded in paraffin, cut into $5-\mu \mathrm{m}$ sections at $100-^{\circ} \mathrm{m}$ intervals throughout the entire sample, and stained with hematoxylin and eosin. A pathologist with no knowledge of the spectroscopic results visually estimated the area \% representing cancer cells, normal epithelial cells, and stroma in each cross-section to the nearest $5 \%$. The volume $\%$ of these features was calculated from the corresponding area $\%$ of each pathological feature on the different cross-sections.

\section{Statistical Analysis}

The 27 most intense resonance peaks/regions of specific metabolites were selected if their means were above the median of all peaks/regions analyzed and were subjected to principal component analysis (PCA). We observed that the first nine principal components (PC1PC9) represented more than $85 \%$ of the total variability for the entire data set; these PCs were included in further analysis.

We examined these nine PCs for their correlations with the amount (volume \%) of epithelium, cancer, and stroma measured from quantitative pathology based on regression analysis to differentiate BCR from non-recurrent clinical-stage-matched groups according to Student's $t$-test. Canonical analyses were performed on four pathologically identified PCs, as well as on the first nine PCs, to discover discriminants that can differentiate BCR from their non-recurrent clinical-stage-matched counterparts. The resulting discriminants in the form of linear combinations of the tested PCs were considered potential BCR metabolomic profiles.

We then applied these defined metabolomic profiles to the pathological-stage-matched control group. To achieve this, the overall loading factors combining coefficients of both PCA and canonical analysis and obtained with standardized metabolite intensities from clinical-stage-matched groups with and without BCR were applied to the standardized metabolite intensities of pathological-stage-matched groups with and without BCR and tested with ANOVA and receiver-operating characteristic (ROC) curve analyses. Statistical analyses were conducted with commercial program JMP 7.0 (SAS Institute, Inc., Cary, NC). 


\section{RESULTS}

\section{High-Resolution Magic Angle Spinning Proton Magnetic Resonance Spectroscopy and Prostate Cancer Pathology}

HRMAS ${ }^{1}$ HMRS allows us to study chemical metabolites in their native states and record intact tissue spectra while preserving the tissue structures for subsequent histopathological analysis. In this study, 11 of 79 analyzed samples from 48 PCa patients contained cancerous glands, whereas the rest $(n=68)$ represented histologically benign tissue obtained from cancerous prostates. Regression analyses on the first nine PCs obtained from clinical-stagematched groups with and without BCR, suggested PC4, 6, and 7 as having statistically significant correlations with quantitative pathologies, while PC8 presented near-significant value in differentiating BCR group from its clinical-stage-matched non-BCR group, as summarized in Table II.

\section{Differentiations Between BCR and Matching Groups}

Pathological processes, which affect more than just a single metabolite, cause a cascade of simultaneous changes in many metabolic pathways and result in alterations in the metabolomic profiles. These profiles can be established through data statistical evaluations including principal component and discriminant analysis to identify the relevant combinations of the measured metabolites that reflect the pathological processes [15].

Figure 1 summarizes the differentiation potentials of metabolomic profiles obtained from canonical analysis performed on PCs obtained from clinical-stage-matched cases, and then applied to the pathological-stage-matched group. In this figure, metabolomic profiles shown as canonical score 1 represent maximal discriminations between clinical-stage-matched groups with and without BCR. The profiles calculated from linear combinations of four histopathologically identified PCs (PC4, 6, 7, and 8) and of all first nine PCs (PC1-9) are shown in Figure 1a and c, respectively. While these two plots obtained from discriminant analyses cannot prove their utility in differentiating BCR from non-recurrence between clinical-stage-matched groups, they suggest the possibility of the existence of such profiles and structures that may be tested with other clinical cases.

To test these profiles, we applied the constructive structures of these profiles onto the experimental data of another paired group that matched patients with and without BCR according to their pathological stages. The evident differentiations between the matched groups for four and the first nine PCs are shown in Figure $1 \mathrm{~b}$ and d, respectively.

Canonical analyses performed on the first nine PCs included more spectral variations than those represented in the four PCs selected from them. Therefore, it is not surprising to observe that the levels of statistical significance presented in Figure 1d with the first nine PCs is much higher than that in Figure 1b. However, examinations of the overall loading coefficients for the evaluated metabolites obtained from nine PCs shown in Figure 1d reveal their linear correlations with the overall coefficients obtained from the analyses of the four pathologically identified PCs, as shown Figure 2. This correlation suggests that although each individual PC among these nine does not present correlations with tissue pathology, the metabolomic profiles obtained from the first nine PCs are still valuable. In addition, examinations of these overall loading coefficients further revealed that the major contributing factors to the observed metabolomic profiles were the changes in metabolites related to PCa developments, including spermine/polyamines, glutamine, myo-inositol, phosphoryl choline, scylloinositol, and glutamate. As shown in Figure 3, the overall accuracies for predicting BCR with the pathological-stage-matched groups based on metabolomic profiles established through analyses of clinical-stage-matched groups for four and nine PCs are $71.1 \%$ and $78.1 \%$, respectively. 


\section{DISCUSSION}

Prediction of BCR after prostatectomy has extremely important clinical significance and has been a topic of extensive clinical investigation $[6-9,16]$. Nevertheless, these studies are empirical in nature and utilize clinical (serum PSA levels, patient ages, etc.) and pathological (cancer Gleason scores, stages, etc.) parameters to construct the prediction models. Thus, for cases with similar clinical and pathological parameters as in the matched groups included in the current study, the empirical models lose their predicting powers differentiating the matched pairs with and without BCR. This retrospective test-of-concept study suggested that among these matched pairs where the existing nomograms are nonfunctional, the measured metabolomic profiles may serve to deliver further differentiation based on tissue cellular biochemical differences. Although the accuracies previously presented are in the range of $70-80 \%$ and further studies of additional cases may improve the sensitivity and specificity of BCR metabolomic profiles, metabolomic profiles may still present a great advancement in a critical clinical situation where prediction of BCR after prostatectomy is non-existent.

Furthermore, the design of the study randomly selecting control groups to match BCR cases first according to clinical and then to pathological stages shows the capability of BCR identifications at different clinical treatment periods from biopsy to prostatectomy. In other words, PCa clinical stages are defined at the time of cancer-positive biopsy prior to prostatectomy, so metabolomic profiles obtained through MRS analysis of biopsy tissue may allow us to identify PCa cases that will likely develop BCR after prostatectomy. Information with such critical clinical relevance is unfortunately unattainable in the current $\mathrm{PCa}$ practice. Therefore, if proven, this protocol may significantly alter the current $\mathrm{PCa}$ treatment paradigm by either preventing prostatectomies that cannot deliver curative therapy but have great risk of surgery-related side-effects, or administrating more aggressive adjuvant therapies after prostatectomy.

However, PCa recurrence may take years to develop. Thus, among the cases we have followed, there are only a limited number of BCR cases available to our study. We recognize the limitation of the present analyses in which we first paired the BCR cases with their clinical-stage-matched cases to discover metabolomic profiles with differentiating powers to be tested on another control group that matched these BCR cases according to the pathological stages. While this arrangement is not an ideal and traditional design of "training" versus "testing" cohorts, the aim of the report is not to draw firm conclusions but rather to inspire conclusions further clinical investigations in this direction.

\section{CONCLUSIONS}

In the current retrospective study, we used intact tissue HRMAS ${ }^{1} \mathrm{HMRS}$ results to generate PCa metabolomic profiles that show potential to predict the probability of postprostatectomy cancer recurrence indicated by the rise of serum PSA. Our results suggest that prostate metabolomic profiles measured with intact tissue HRMAS ${ }^{1} \mathrm{HMRS}$ are capable of providing an additional parameter to predict the risk of BCR beyond the capacity of the current predicting nomograph used in clinic and the current knowledge of pathology. With additional studies in this direction, PCa metabolomic profiles may provide a valuable adjunct biochemical parameter that will contribute significantly not only to saving the lives of PCa patients but also to preserving their quality of life.

\section{Acknowledgments}

This work was supported by CA115746 and CA095624 (to L.L.C.), Bertucci Prostate Cancer Fund (C.-L.W.), and MGH A. A. Martinos Center for Biomedical Imaging. 


\section{REFERENCES}

1. Jemal A, Siegel R, Ward E, Hao Y, Xu J, Murray T, Thun MJ. Cancer statistics, 2008. CA Cancer J Clin. 2008; 58:71-96. [PubMed: 18287387]

2. Andriole GL, Crawford ED, Grubb RL III, Buys SS, Chia D, Church TR, Fouad MN, Gelmann EP, Kvale PA, Reding DJ, Weissfeld JL, Yokochi LA, O’Brien B, Clapp JD, Rathmell JM, Riley TL, Hayes RB, Kramer BS, Izmirlian G, Miller AB, Pinsky PF, Prorok PC, Gohagan JK, Berg CD. Mortality results from a randomized prostate-cancer screening trial. N Engl J Med. 2009; 360(13): 1310-1319. [PubMed: 19297565]

3. Schroder FH, Hugosson J, Roobol MJ, Tammela TL, Ciatto S, Nelen V, Kwiatkowski M, Lujan M, Lilja H, Zappa M, Denis LJ, Recker F, Berenguer A, Maattanen L, Bangma CH, Aus G, Villers A, Rebillard X, van der Kwast T, Blijenberg BG, Moss SM, de Koning HJ, Auvinen A. Screening and prostate-cancer mortality in a randomized European study. N Engl J Med. 2009; 360(13):13201328. [PubMed: 19297566]

4. Shariat SF, Karakiewicz PI, Suardi N, Kattan MW. Comparison of nomograms with other methods for predicting outcomes in prostate cancer: A critical analysis of the literature. Clin Cancer Res. 2008; 14(14):4400-4407. [PubMed: 18628454]

5. Shariat SF, Karakiewicz PI, Margulis V, Kattan MW. Inventory of prostate cancer predictive tools. Curr Opin Urol. 2008; 18(3):279-296. [PubMed: 18382238]

6. Karakiewicz PI, Hutterer GC. Predictive models and prostate cancer. Nat Clin Pract Urol. 2008; 5(2):82-92. [PubMed: 18259186]

7. Shariat SF, Walz J, Roehrborn CG, Zlotta AR, Perrotte P, Suardi N, Saad F, Karakiewicz PI. External validation of a biomarker-based preoperative nomogram predicts biochemical recurrence after radical prostatectomy. J Clin Oncol. 2008; 26(9):1526-1531. [PubMed: 18349404]

8. Steuber T, O’Brien MF, Lilja H. Serum markers for prostate cancer: A rational approach to the literature. Eur Urol. 2008; 54(1):31-40. [PubMed: 18243505]

9. Makarov DV, Trock BJ, Humphreys EB, Mangold LA, Walsh PC, Epstein JI, Partin AW. Updated nomogram to predict pathologic stage of prostate cancer given prostate-specific antigen level, clinical stage, and biopsy Gleason score (Partin tables) based on cases from 2000 to 2005 . Urology. 2007; 69(6):1095-1101. [PubMed: 17572194]

10. Hara R, Jo Y, Fujii T, Kondo N, Yokoyoma T, Miyaji Y, Nagai A. Optimal approach for prostate cancer detection as initial biopsy: Prospective randomized study comparing transperineal versus transrectal systematic 12-core biopsy. Urology. 2008; 71(2):191-195. [PubMed: 18308081]

11. Wright JL, Lange PH. Newer potential biomarkers in prostate cancer. Rev Urol. 2007; 9(4):207213. [PubMed: 18231617]

12. Lilja H, Ulmert D, Vickers AJ. Prostate-specific antigen and prostate cancer: Prediction, detection and monitoring. Nat Rev Cancer. 2008; 8(4):268-278. [PubMed: 18337732]

13. Cheng LL, Burns MA, Taylor JL, He W, Halpern EF, McDougal WS, Wu CL. Metabolic characterization of human prostate cancer with tissue magnetic resonance spectroscopy. Cancer Res. 2005; 65(8):3030-3034. [PubMed: 15833828]

14. Taylor JL, Wu CL, Cory D, Gonzalez RG, Bielecki A, Cheng LL. High-resolution magic angle spinning proton NMR analysis of human prostate tissue with slow spinning rates. Magn Reson Med. 2003; 50(3):627-632. [PubMed: 12939772]

15. Cheng, LL.; Pohl, U. The role of NMR-based metabolomics in cancer. In: Lindon, JC.; Nicholls, JK.; Holmes, E., editors. The handbook of metabonomics and metabolomics. Amsterdam: Elsevier; 2007. p. 345-374.

16. Scher, HI.; Leibel, SA.; Fuks, Z.; Cordon-Cardo, C.; Scardino, PT. Cancer of the prostate. In: DeVita, V.; Hellman, S.; Rosenberg, S., editors. Cancer: Principle and practice of oncology. 7th edition. Philadelphia: Lippincott Williams \& Wilkins; 2005. p. 1192-1259. 


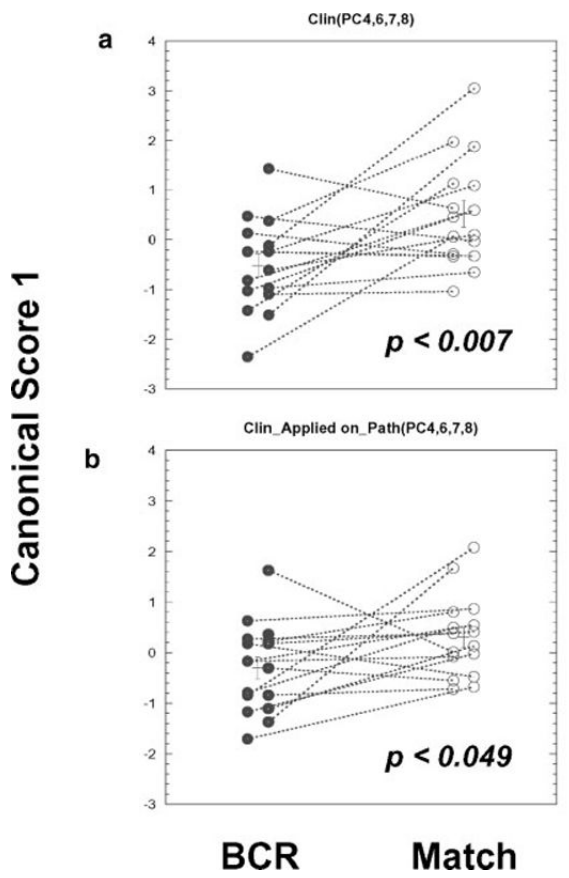

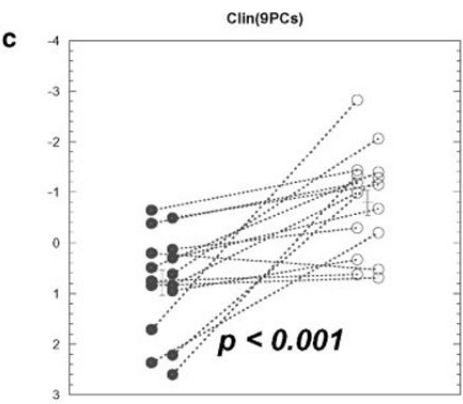



Fig. 1.

Metabolomic profiles differentiate prostate cancer recurrence from clinical- and pathological-stage-matched cases. Metabolomic profiles calculated from intact tissue proton MRS measured at 14T after prostatectomy can differentiate cases of PCa biochemical recurrence $(\mathrm{BCR}, \mathrm{n}=16$, closed dots) from clinical- and pathological-stage-matched cases $(\mathrm{n}=16$ each, open dots) without BCR. These 16 BCR cases represent all the BCR cases among a pool of 183 prostatectomy cases that we analyzed between 2002 and 2003. The matched cases without BCR were also randomly selected from this pool. Metabolomic profiles that can maximally differentiate between clinical-stage-matched groups with and without BCR were obtained from principal component analyses and canonical analysis for (a) four histopathologically identified PCs (PC4, 6, 7, and 8), and for (c) the first nine PCs (PC1-9). By applying the combination structures of these profiles to another paired group that matched patients with and without BCR according to their pathological stages, the differentiation power persists as shown for (b) the selected four and (d) the first nine PCs. The levels of statistical significances are listed in the plots. 


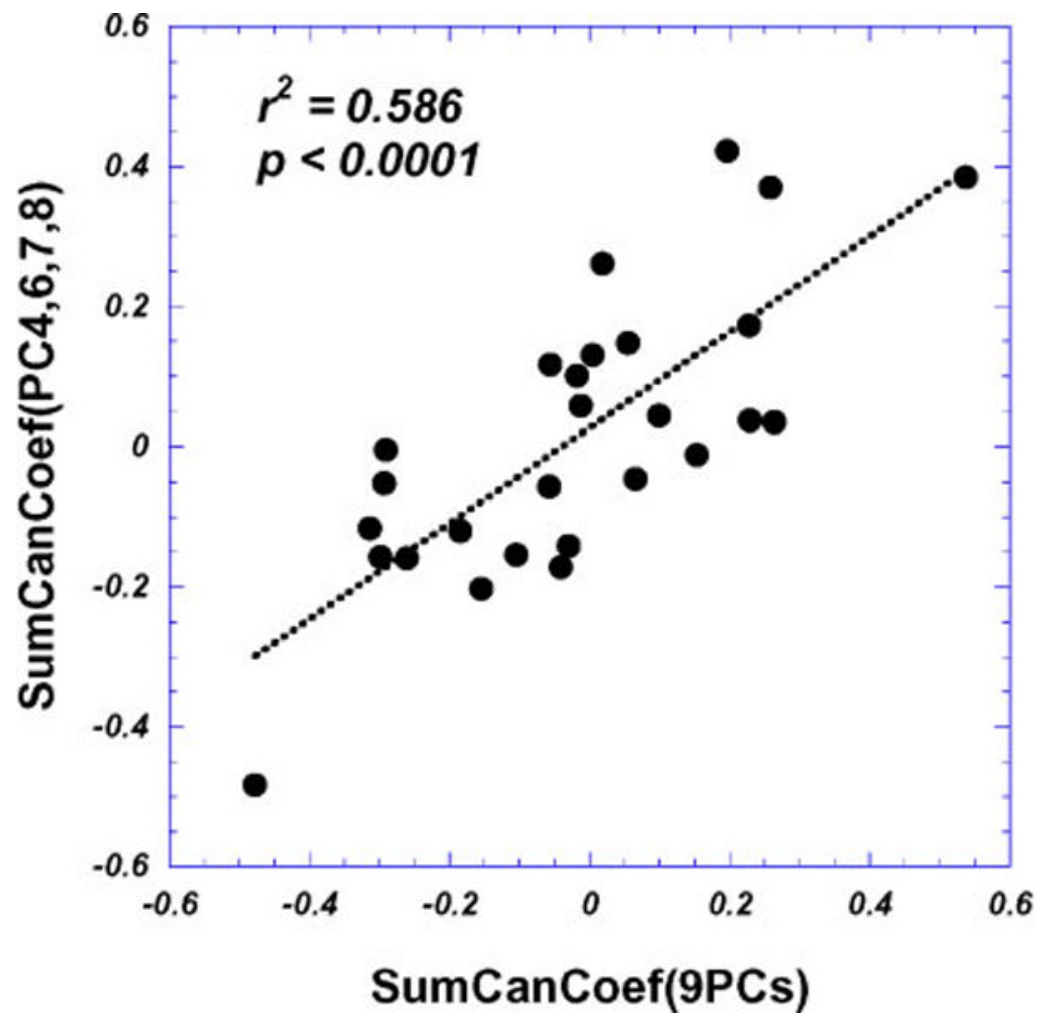

Fig. 2.

Linear correlation between metabolomic profiles calculated from four pathologically identified PCs and the first nine PCs. The comparison of the sums of the overall coefficients (SumCanCoef), combinations of coefficients from both principal component analysis of standardized spectral intensities and canonical analysis of relevant PCs reveals a linear relationship between the overall coefficients obtained with PC4, 6, 7, and 8 and with the first nine PCs. This correlation validates the utility of metabolomic profiles obtained from the first nine PCs, although not each individual PC presents a correlation with tissue pathology. 
PC4, 6, 7, \& 8

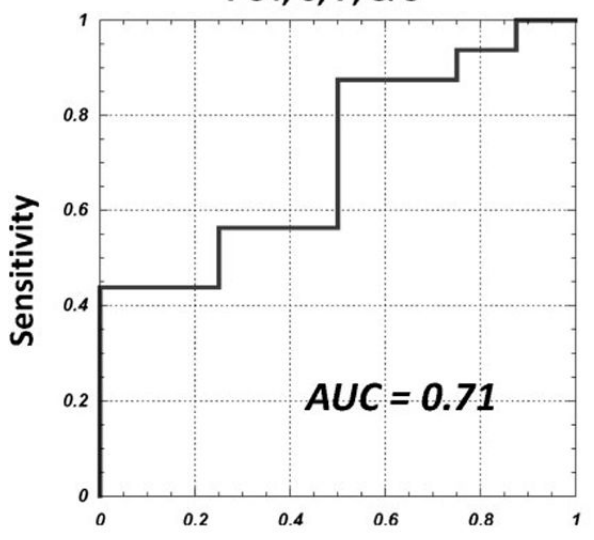

PC1-9

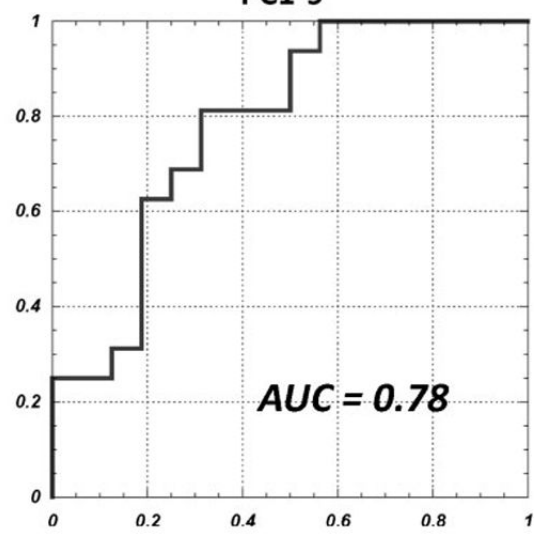

1 - Specificity

Fig. 3.

The overall accuracies in identifying BCR from pathological-stage-matched cases according to metabolomic profiles calculated from clinical-stage-matched cases. Receiver-operating characteristic (ROC) curves for identifying BCR from pathological-stage-matched non-BCR cases used as a "testing-set" revealed overall accuracies of $71 \%$ and $78 \%$ for four (Fig.1b) and the first nine (Fig.1d) PCs, respectively. This indicates the utility of PCa metabolomic profiles sensitive to BCR obtained from the "training-set" of clinical-stage-matched cases with and without BCR. 







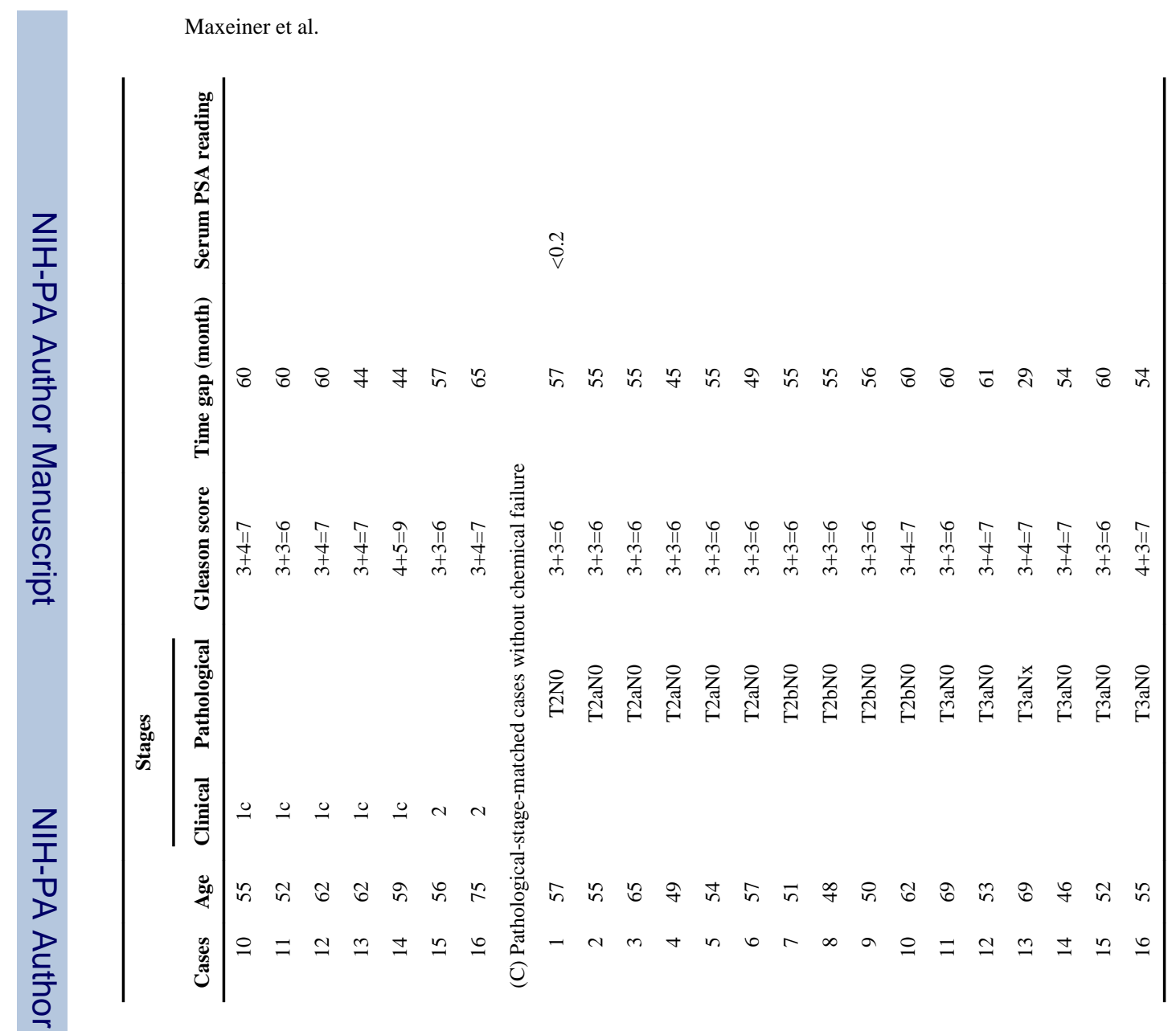

Page 11 
TABLE II

Principal Components and Their Correlations With Prostate Pathologies

\begin{tabular}{lcccc}
\hline & PC4 & PC6 & PC7 & PC8 \\
\hline Eigenvalue & 1.760 & 1.394 & 1.240 & 1.026 \\
$\%$ & 6.52 & 5.16 & 12 & 3.80 \\
Cumulative \% & 63.91 & 74.81 & 79.40 & 83.21 \\
Benign (vol. \%) & & $\mathrm{r}=-0.360, P<0.043$ & $\mathrm{r}=+0.355, P<0.046$ & $t$-test: $P=0.054$ \\
Cancer (vol. \%) & $\mathrm{r}=+0.447, P<0.010$ & $\mathrm{r}=+0.430, P<0.014$ & & \\
\hline
\end{tabular}

